\title{
CONSEJO DE ADMINISTRACIÓN Y DESEMPEÑO FINANCIERO. UN ESTUDIO BIBLIOMÉTRICO
}

\section{BOARD OF DIRECTORS AND FINANCIAL PERFORMANCE. A BIBLIOMETRIC STUDY}

\section{CONSELHO DE ADMINISTRAÇÃO E DESEMPENHO FINANCEIRO. UM ESTUDO BIBLIOMÉTRICO}

Luis Améstica-Rivas; Andrea King-Domínguez; Carlos Cruzat Valenzuela;

Constanza Stuardo Solar

Doctor en Administración y Dirección de Empresas, Universidad Politécnica de Cataluña. Académico Departamento de Gestión Empresarial, FACE, Universidad del Bío-Bío. ORCiD: 0000-0003-0482-0287. E-mail: lamestica@ubiobio.cl, Chile.

Magíster en Administración mención Gestión Financiera, Universidad de Chile. Académica Depto. de Economía y Finanzas, FACE, Universidad del Bío-Bío. ORCiD: 0000-0002-1063-4336. E-mail: aking@ubiobio.cl, Chile.

Licenciado en Ciencias Administrativas, Universidad del Bío-Bío. Programa Magíster en Gestión de Empresas (MGE), Universidad del Bío-Bío. ORCiD: 0000-0002-5495-7313. E-mail: carlos.cruzat1501@alumnos.ubiobio.cl, Chile.

Licenciada en Ciencias Administrativas, Universidad del Bío-Bío. Programa Magíster en Gestión de Empresas (MGE), Universidad del Bío-Bío. ORCiD: 0000-0001-6384-6191. E-mail: constanza.stuardo1501@alumnos.ubiobio.cl, Chile.

Recibido: 23 de mayo de 2020

Aprobado: 28 de septiembre de 2020

DOI: https://doi.org/10.22267/rtend.212201.160 


\title{
Resumen
}

Este trabajo analiza la evolución que ha tenido la investigación científica sobre los "consejos de administración" o llamados "directorios" y su relación con los "resultados financieros de las empresas”. Se propone un análisis bibliométrico con base a revistas indexadas en Web of Science (WoS) hasta el año 2019, permitiendo evidenciar la relevancia que ha tenido para las empresas y el mundo científico. Metodológicamente, se analizó inicialmente 4.468 artículos del área de negocios, filtrando la búsqueda de al menos una de las palabras clave, encontrando 3.302 publicaciones desde 1988. Se aplicaron técnicas bibliométricas apoyadas por el software VOSviewer. Entre los resultados se destaca que las publicaciones se concentran en 637 revistas, con la mayor producción entre el 2009 y 2019, equivalente al 80,8\% del total. Tres revistas de alto impacto concentran la mayor cantidad de publicaciones. Europa lidera la producción científica, destacando la académica Isabel María García Sánchez y el autor más citado David Yermack. Las palabras clave más citadas son gobierno corporativo, directores y desempeño. Se observan nuevas líneas potenciales de investigación en temas de diversidad, mujeres y características propias en los CA como el estudio de caso de países emergentes y empresas familiares.

Palabras clave: bibliométrico; consejo de administración; desempeño financiero; gestión; empresa.

JEL: G3; G32; G34; M10; M19

\begin{abstract}
This work analyzes the evolution of scientific research on "boards of directors" or so-called "directories" and their relationship with "financial results of companies". It proposes a bibliometric analysis based on magazines indexed in the Web of Science (WoS) until the year 2019, allowing to evidence the relevance it has had for companies and the scientific world. Methodologically, 4,468 articles of the business area were initially analyzed, filtering the search of at least one of the key words, finding 3,302 publications since 1988. Bibliometric techniques supported by the VOSviewer software were applied. Among the results, it stands out that the publications are concentrated in 637 magazines, with the highest production between 2009 and 2019, equivalent to $80.8 \%$ of the total. Three high impact journals concentrate the largest number of publications. Europe leads the scientific production, standing out the academic Isabel María García Sánchez and the most quoted author David Yermack. The most cited key words are corporate governance, directors and performance. New potential lines of research on diversity, women, and characteristics of CA are observed, such as the case study of emerging countries and family businesses.
\end{abstract}


Keywords: bibliometrics; board of directors; firm performance; management; company.

JEL: G3; G32; G34; M10; M19

\section{Resumo}

Este artigo analisa a evolução da investigação científica sobre "conselhos de administração" ou os chamados "directórios" e a sua relação com os "resultados financeiros das empresas". Propõe uma análise bibliométrica baseada em revistas indexadas na Web of Science (WoS) até 2019, permitindo evidenciar a relevância que teve para as empresas e o mundo científico. Metodologicamente, 4.468 artigos da área de negócios foram inicialmente analisados através da filtragem da pesquisa de pelo menos uma das palavras-chave, encontrando 3.302 publicações desde 1988. Foram aplicadas técnicas bibliométricas suportadas pelo software VOSviewer. Entre os resultados, é de notar que as publicações estão concentradas em 637 revistas, com a maior produção entre 2009 e 2019, o equivalente a 80,8\% do total. Três revistas de grande impacto concentram o maior número de publicações. A Europa lidera a produção científica, destacando-se a académica Isabel María García Sánchez e o autor mais citado David Yermack. As palavras-chave mais citadas são corporate governance, directores e desempenho. São observadas novas linhas potenciais de investigação sobre questões de diversidade, mulheres e características da AC, tais como o estudo de casos de países emergentes e empresas familiares.

Palavras-chave: bibliometria; conselhos de administração; desempenho financeiro; administração; empresa.

JEL: G3; G32; G34; M10; M19

\section{Introducción}

El Consejo de Administración (CA) cumple un rol primordial en las organizaciones, siendo un factor determinante de su funcionamiento. Es el encargado de gestionar o determinar las estrategias necesarias a seguir por la compañía para que esta obtenga un buen desempeño a lo largo del tiempo, preocupándose de la relación entre el poder decisión y el valor creado. En este contexto, se puede decir que cada vez son más los estudios que hablan sobre la correspondencia entre la toma de decisiones y el desempeño financiero o el resultado empresarial. En la mayoría de las investigaciones se establece una relación positiva entre estas dos variables, es decir, que las resoluciones tomadas por el CA repercuten en el resultado financiero de la firma. 
En su definición más básica el CA o llamado indistintamente en alguna literatura directorio, es el máximo órgano colegiado responsable del gobierno corporativo; de su administración, control, dirección y supervisión de la compañía, donde sus miembros deben ser elegidos o ratificados por la junta de accionistas. Por ende, la supervivencia de la organización depende, en gran parte, de la forma como administra su poder. Asimismo, el CA es uno de los principales mecanismos para alinear los intereses de los accionistas y gerentes en el sistema de gobierno (Fama y Jensen, 1983), base fundamental de la teoría de agencia.

En tanto, el desempeño financiero está vinculado directamente con características propias del directorio (Al Farooque et al., 2019). Por ejemplo, Yasser et al. (2017), en un estudio realizado a compañías de Pakistán, examinan la relación entre la demografía de la junta y el desempeño financiero de estas, describiendo los atributos y/o características de cada una y sus CA. Entre los resultados, se evidencia una relación positiva entre el tamaño de la junta, la representación minoritaria en esta y el desempeño interno, como director de familia propietaria. Sin embargo, encuentran que los directores independientes están negativamente asociados con el valor de la empresa.

Por otro lado, el CA tiene directa relación con la estrategia empresarial (Patel, 2019). El directorio generalmente influye en todas las decisiones relevantes de una organización, incluido el establecimiento de sus objetivos a largo plazo, el desarrollo de una política estratégica corporativa, así como la contratación y fijación de la compensación del director ejecutivo. Además, la forma como se estructura el CA puede tener un efecto significativo en el monitoreo y la gobernanza de la empresa. Este artículo pretende ser un aporte al área, estructurándose desde una introducción, referentes teóricos donde explicita brevemente la evolución que han tenido las técnicas bibliométricas y su utilización para temas de CA en el área de negocios. Luego, se expone la metodología que se aplicó en forma secuencial y la discusión de los resultados. Finalmente, en las conclusiones se señalan las contribuciones más relevantes de la investigación.

\section{Referentes teóricos}

En esta etapa se realiza un análisis histórico de las publicaciones en el área y su relevancia para la gestión. Es términos evolutivos, inicialmente las publicaciones indexadas a la base de datos Web of Science (WoS - ex ISI) en el área del CA y desempeño financiero eran mínimas, con sólo 122 artículos entre 1988 y 1998. Desde los inicios de esta base de datos se encontraron 4.468 artículos. 
En la última década las investigaciones en torno a los conceptos de CA y desempeño financiero aumentaron de manera exponencial, registrándose 2.667 publicaciones en revistas indexadas a la WoS entre los años 2009 y 2019. Considerando la relevancia que ha tomado el tema de CA y su relación con el desempeño en los últimos años, especialmente para el mundo empresarial y las escuelas de negocios, es de interés detectar el estado actual y evolución sobre la materia, innovando en el estudio utilizando para ello técnicas bibliométricas más actuales que permiten un mejor análisis, desde un enfoque mixto.

Según Ferreira y De Morán (2011), la bibliometría ha evolucionado en sus técnicas y herramientas de apoyo, con la incorporación de las matemáticas y los métodos estadísticos a toda fuente escrita y que considere elementos tales como: autores, título de la publicación, tipo de documento, idioma, resumen y palabras claves o descriptores, entre otros. En este sentido, Merediz-Solà y Bariviera (2019), señalan que los análisis bibliométricos ayudan a los nuevos investigadores de una disciplina a comprender el alcance de un tema, las tendencias emergentes y su evolución a través del tiempo.

\section{Metodología}

Para analizar el estado de la investigación sobre CA y desempeño financiero se realizó una exploración a la colección de la Web of Science (WoS), reconocida como una de las herramientas internacionales y multidisciplinaria de mayor reconocimiento para el mundo científico. En virtud de ello y para obtener resultados más precisos se hicieron una serie de filtros. Inicialmente se utilizó la opción "búsqueda avanzada", definiendo cada tema (TS) para cada una de las exploraciones, dejando solo aquellas que pertenecían a artículos, excluyendo libros, conferencias y ponencias. El periodo de estudio se extendió a noviembre de 2019. La realización de la búsqueda se basó en los siguientes criterios, de forma ordenada y enumerada, incorporando las siguientes palabras clave:

- Primera exploración: "directorate*” or "board director*” or "governing council*"

- Segunda exploración: "performance*" or "economic result*" or "financial performance*”

- Tercera exploración: se realizó la unión entre la primera y segunda exploración con la finalidad de generar una base que concatenara las dos dimensiones anteriores. 
En esta etapa, el sistema arrojó 4.468 artículos publicados en las diferentes categorías. Cada artículo fue revisado con la finalidad de verificar la presencia de las palabras claves definidas en las dos exploraciones iniciales y que tuvieran coherencia con la temática central. El paso siguiente fue una refinación de las categorías ofrecidas por la plataforma, considerando solo aquellas correspondientes al área de negocios, debido al carácter y rama investigativa. A partir de esta etapa, se depuró en las categorías "management" or "business" or "business finance" or "economics", obteniendo un resultado de 3.302 artículos presentes entre los años 1988 y 2019, haciendo una segunda revisión de títulos, resúmenes y palabras clave.

Finalmente, con los datos obtenidos y con el apoyo de la planilla electrónica Excel y software VOSviewer $^{1}$ se comienza un nuevo análisis. Se vaciaron en Excel y como archivo texto, ello permitió la aplicación del software VOSviewer, el cual permite crear mapas de datos, facilitando una mejor visualización y exploración (King-Domínguez et al., 2020; Van Eck y Waltman, 2017). Dado el procedimiento descrito, se construyó un archivo base en donde se generó un análisis evolutivo a lo largo de los años. El estudio es de carácter descriptivo y este permite distinguir el número de artículos, autores, revistas y organizaciones para cada año como a su vez el número de referencias utilizadas dentro de los periodos seleccionados. Para una visión más clara, se establecieron periodos de análisis con rangos de 10 años, considerando el primer artículo publicado en 1988 hasta 2019. A partir de la distinción de los autores seminales y tendenciales, se calcularon indicadores bibliométricos básicos referentes a distintas métricas, como por ejemplo: número de autores por artículo, por revista y por organización.

En una segunda etapa contempló un enfoque más cuantitativo, con el objetivo de generar un análisis a nivel de detalle. En primera instancia, las revistas se ordenaron de forma decreciente debido al gran número de artículos. Luego, en segunda instancia, se determinó los principales autores, para ello se revisó el número de citas recibidas y número de artículos publicados a la fecha. De la misma manera, se definieron las redes de trabajos entre los autores, considerando aquellos que han publicado como mínimo ocho documentos, parámetro que se definió a partir del universo de publicaciones en el área.

En una tercera instancia, se determinaron las organizaciones que investigan sobre la temática, dado por el número de artículos publicados. De modo complementario se determinó la colaboración

\footnotetext{
${ }^{1}$ VOSviewer es una herramienta de software para crear, visualizar y explorar mapas basados en datos de red.
} 
existente entre ellas y el país de origen. Lo anterior fue realizado con el software VOSviewer, tomando como criterio las organizaciones que tenían al menos cinco publicaciones.

En una cuarta instancia y en virtud de lo planteado por Huang y Ho (2011), "el objetivo de descubrir tendencias y patrones, se midió la potencia en la asociación de términos representativos de las publicaciones, estudiando las palabras clave utilizadas por los diferentes autores, lo cual facilita entender los temas que son abordados por los investigadores principales". Para ello, se realizó un análisis de las palabras clave utilizadas por los autores sugeridos por WoS. Complementariamente y tomando las indicaciones de King-Domínguez et al. (2020), se abordó el uso de las "palabras clave" y "co-palabras", con un mínimo de ochos ocurrencias. A su vez, para una mejor interpretación de resultados, se generó los clúster (conglomerado), conformadas con las palabras más importantes, incluyendo 18 palabras claves para cada uno de los primeros cuatro clústeres y 11 palabras claves para el último.

De forma final, para conocer los elementos sobre los cuales se ha investigado sobre directorio y desempeño financiero, se estudiaron las referencias más ocupadas en los estudios.

\section{Discusión}

En concordancia con lo anterior, en este apartado se realiza un análisis y discusión a nivel de detalle de las características que presentan los estudios sobre CA y desempeño financiero.

\section{Evolución de artículos y revistas sobre CA y desempeño financiero}

Según la base de datos WoS, las publicaciones sobre CA y desempeño financiero comenzaron en el año 1988. Desde esa fecha, la producción científica fue esporádica, llegando a 27 artículos en el año 2002. A partir del año 2003 la literatura en este campo aumento de manera significativa, principalmente en última década, publicándose 2.667 artículos en el período 2009-2019, equivalente al 80,8\% del total (Figura 1). 


\section{Figura 1}

Evolución de los artículos y revistas sobre CA y desempeño financiero

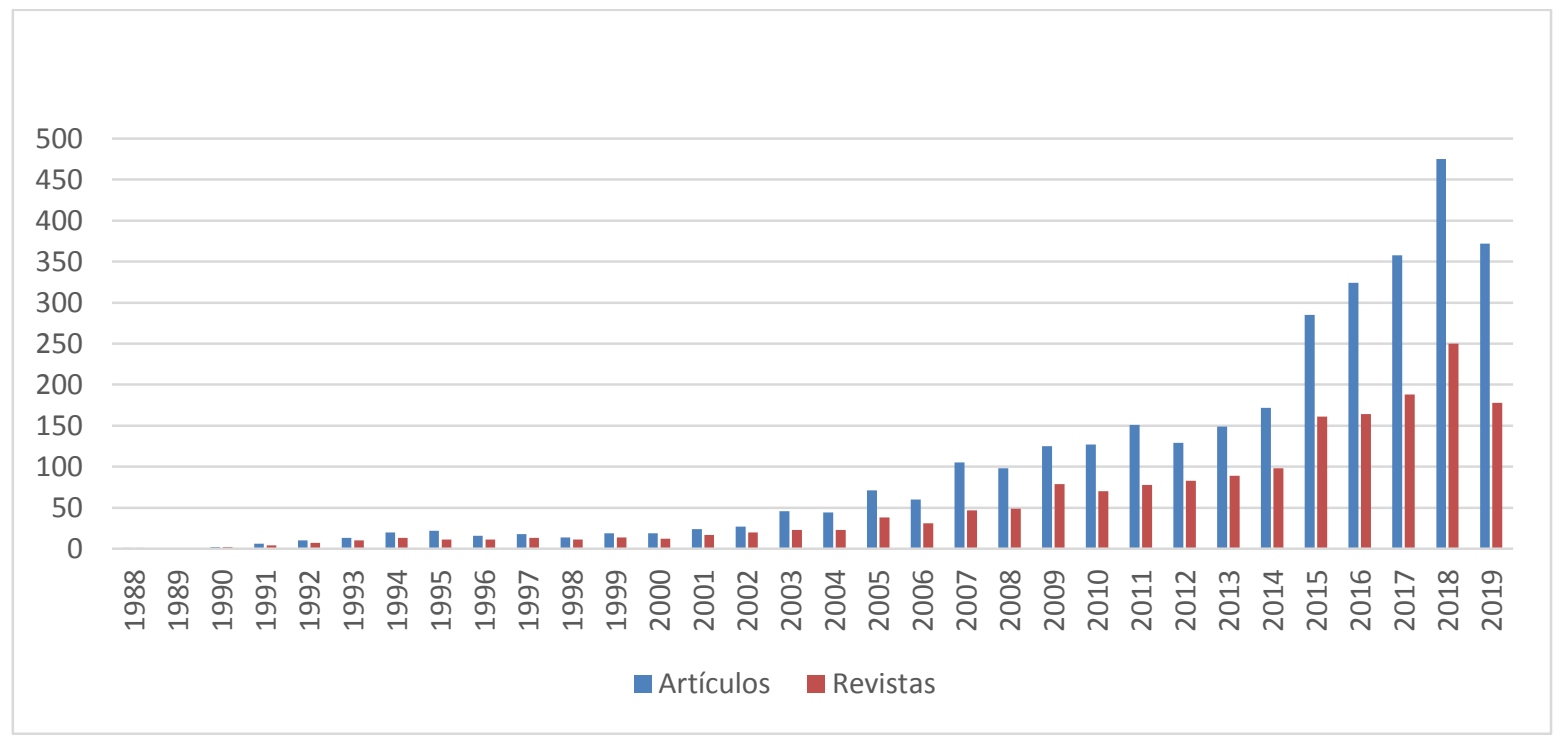

Fuente: elaboración propia.

Los artículos referentes al área investigada han ido creciendo junto a otros indicadores, como el número de autores, revistas y organizaciones (véase Tabla 1). Con respecto a las revistas, se observa que 637 han publicado artículos sobre la temática con 3.302 artículos. En un primer periodo (1988-1998), el número de revistas alcanzó a 38 distribuido en 122 artículos, estableciendo una relación de artículos por revista de 3,2. En el segundo y tercer período esta relación aumento a 4,1 y 4,5 respectivamente. Es decir, gran parte de estas fuentes han publicado más de una investigación, alcanzando una relación artículos por revista desde 1988 de 5,1 y con un indicador de 1,7 autores por artículo en promedio. Asimismo, destaca la cantidad de autores por organización que llega a 2,5. Por otro lado, es importante mencionar que la mayor producción de los artículos (2.667) fue publicada entre 2009-2019, periodo en que se releva la importancia del tema, con una producción creciente cada año.

\section{Tabla 1}

Caracterización de las publicaciones en directorio y desempeño financiero

\begin{tabular}{lrrrr}
\hline & $1988-1998$ & $1999-2008$ & $2009-2019$ & \multicolumn{1}{c}{ Total } \\
\hline $\mathrm{N}^{\circ}$ de artículos & 122 & 513 & 2.667 & 3.302 \\
$\mathrm{~N}^{\circ}$ de autores & 189 & 892 & 4.829 & 5.568 \\
$\mathrm{~N}^{\circ}$ de revistas & 38 & 125 & 590 & 637 \\
$\mathrm{~N}^{\circ}$ de organizaciones & 111 & 464 & 2.043 & 2.212
\end{tabular}




$\begin{array}{lrrrr}\mathrm{N}^{\circ} \text { de referencias } & 3.260 & 13.053 & 68.962 & 70.439 \\ \mathrm{~N}^{\circ} \text { autores por artículo } & 1,5 & 1,7 & 1,8 & 1,7 \\ \mathrm{~N}^{\circ} \text { de artículos por revista } & 3,2 & 4,1 & 4,5 & 5,1 \\ \mathrm{~N}^{\circ} \text { de autores por organización } & 1,7 & 1,9 & 2,4 & 2,5\end{array}$

Fuente: elaboración propia.

\section{Principales revistas}

A través del ordenamiento decreciente del número de publicaciones, se evidencia que las revistas con mayor cantidad de publicaciones son: Corporate Governance an International Review con 215 artículos, Journal of Corporate Finance con 120 y Journal of Business Ethics con 118 artículos, tal como se refleja en la Tabla 2. Al revisar la calidad de las publicaciones medida por el factor de impacto, de las 13 revistas con mayor cantidad de artículos, 11 de ellas $(84,6 \%)$ pertenecen al Quartil I. A excepción de la revista Corporate Governance the International Journal of Business in Society de origen asiático, la mayoría de las revistas son Europeas y de América del Norte, como por ejemplo: Revistas Holandesas; Journal of Corporate Finance, Journal of Business Ethics, Journal of Financial \& Economics, Journal of Banking \& Finance. Del Reino Unido; Management Decision, British Journal of Management, Journal of Business Finance \& Accounting y de Estados Unidos; Accounting and Finance, Academy of Management Journal, Strategic Management Journal, Corporate Governance an International Review. En este mismo sentido y según la tipología por área dada por la propia WoS, las revistas que más publican sobre CA y desempeño financiero lo hacen a través de las categorías: negocios, administración, finanzas y economía.

\section{Tabla 2}

Revistas y publicaciones principales

\begin{tabular}{llccc}
\hline \multicolumn{1}{c}{ Revista } & Categorías WoS & $\begin{array}{c}\text { Factor } \\
\text { de } \\
\text { Impacto }\end{array}$ & $\begin{array}{c}\text { Cuartil } \\
\text { (Q) }\end{array}$ & $\begin{array}{c}\text { Artículos } \\
\text { publicados }\end{array}$ \\
\hline $\begin{array}{llll}\text { Corporate Governance an } \\
\text { International Review }\end{array}$ & Negocios & & & \\
& Administración & 4.408 & 1 & 215 \\
Journal of Corporate Finance & Finanzas & & & \\
& Negocios & 3.184 & 1 & 120
\end{tabular}




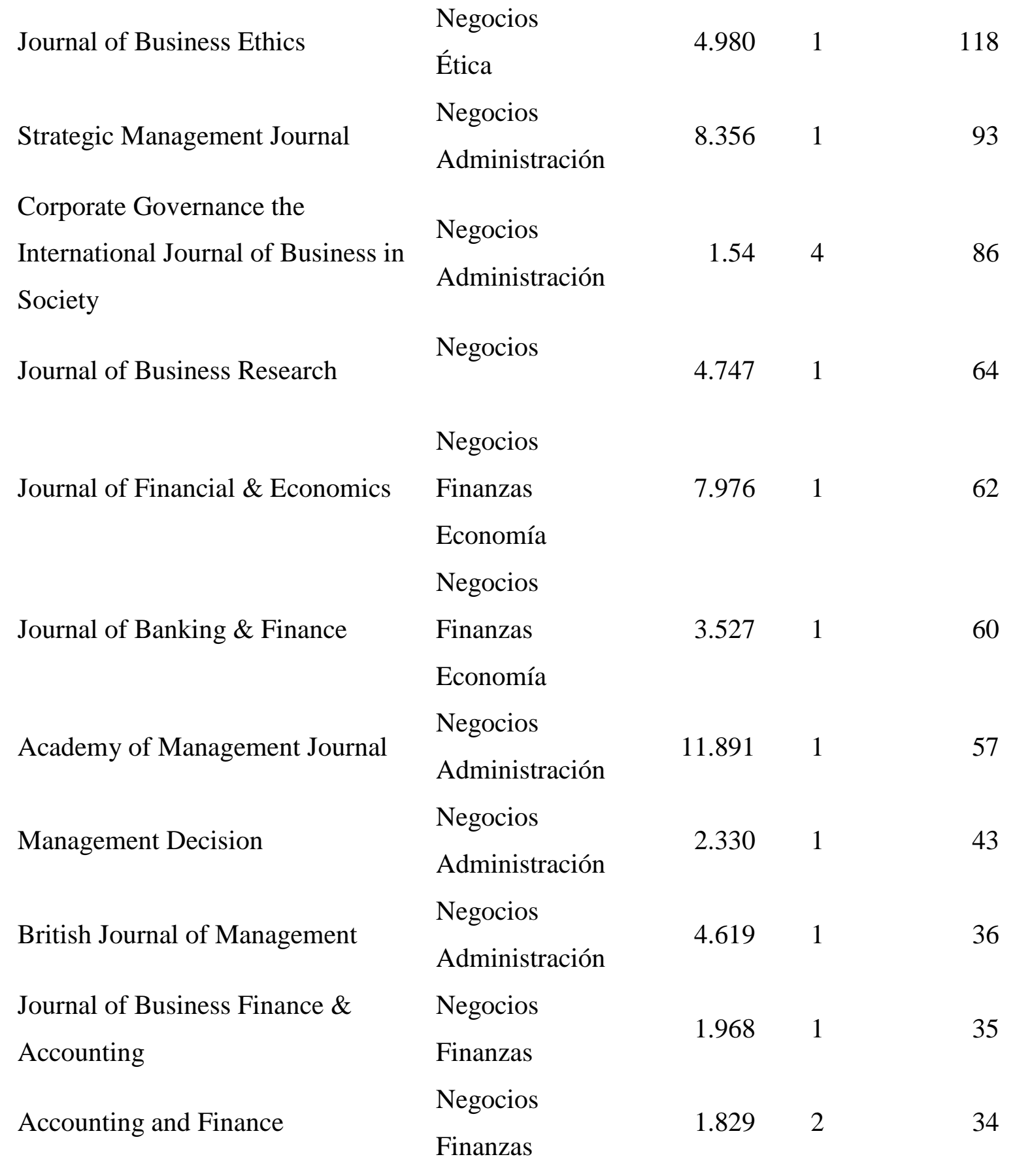

Fuente: elaboración propia.

\section{Organizaciones}

En relación a las organizaciones participantes en las publicaciones, esta alcanza a 2.212 instituciones, destacándose entre ellas Arizona State University con 52 artículos, seguida por Texas A\&M University con 44 y Penn State University con 40 (Tabla 3). Siete organizaciones de Estados Unidos acumulan 313 artículos, liderando la producción científica entre 6 naciones. En Europa, destacan dos instituciones: University of Salamanca de España con 29 y Bocconi 
University de Italia con 28 artículos, respectivamente. China presenta dos instituciones: City University Hong Kong y Hong Kong Polytech University, que sumados alcanzan 54 documentos.

\section{Tabla 3}

Principales organizaciones que publican sobre CA y desempeño financiero

\begin{tabular}{lcl}
\hline \multicolumn{1}{c}{ Institución } & $\mathrm{N}^{\circ}$ de documentos & \multicolumn{1}{c}{ País } \\
\hline Arizona State University & 52 & Estados Unidos \\
Texas A\&M University & 44 & Estados Unidos \\
Penn State University & 40 & Estados Unidos \\
Indiana University & 38 & Estados Unidos \\
University of Illinois & 33 & Estados Unidos \\
Northeastern University & 29 & Estados Unidos \\
University of Salamanca & 29 & España \\
Bocconi University & 28 & Italia \\
City University Hong Kong & 28 & China \\
Massey University & 27 & Nueva Zelanda \\
Queensland University of Technology & 27 & Australia \\
Harvard University & 26 & Estados Unidos \\
Hong Kong Polytech University & 26 & China \\
University of Michigan & 26 & Estados Unidos \\
University of Missouri & 25 & Estados Unidos \\
\hline
\end{tabular}

Fuente: elaboración propia.

A partir del análisis realizado sobre aquellas instituciones que mayor aporte, se determinó el nivel de colaboración que existe entre ellas. Y tal como señaló en la metodología, se utilizó el software VOSviewer, obteniendo tres grupos (Figura 2) de instituciones colaborativas. El mapa muestra un primer clúster (verde), compuesto por 118 instituciones. En ellas, destaca la participación de: Universidad Estatal de Georgia (249), Universidad de la Ciudad de Hong Kong (217), Universidad de Lehigh (204), Universidad Rutgers (196) y Universidad de Queensland (195). El segundo clúster (azul) estaría formado 84 instituciones, destacando cinco como las más relevantes: Universidad Estatal de Arizona (286), Universidad de Texas A\&M (265), Universidad de Misuri (262), Universidad de Illinois (233) y la Universidad de Indiana (226). Finalmente, en el tercer clúster (rojo) participan 114 instituciones. De estas últimas, las que han participado con mayor 
cantidad de artículos son: Universidad de Míchigan (260), Universidad de Pensilvania (259), Universidad de Nueva York (241), Universidad de Arizona (227) y Universidad de Nueva Gales del Sur (214).

\section{Figura 2}

\section{Colaboración entre organizaciones}

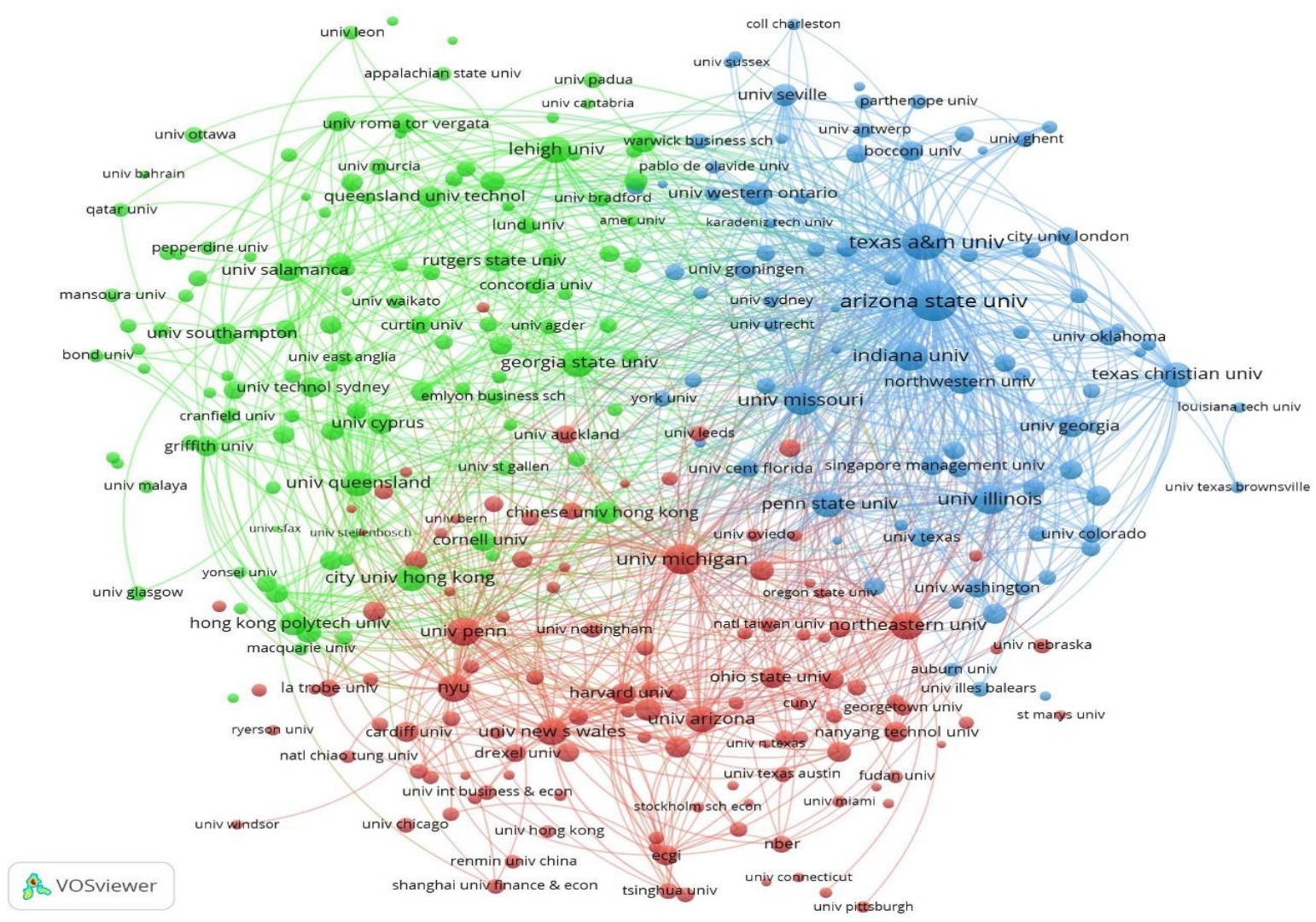

Fuente: elaboración propia a partir de VOSviewer.

\section{Productividad científica según países e idioma de la publicación}

Si se realiza un análisis geográfico, se puede evidenciar que las publicaciones principales se concentran en el continente Europeo con 1.599 artículos $(48,4 \%)$, luego en forma decreciente lo siguen América con 1466 artículos (44,4\%), Asia con 985 artículos (29,8\%), Oceanía con 383 artículos $(11,6 \%)$ y finalmente, África con 128 artículos (3,9\%). En cuanto a los países, en América destaca Estados Unidos con 1.225 artículos (37,1\%). En Europa sobresalen: Inglaterra con $353(10,7 \%)$ y España con 262 documentos (7,9\%). En Oceanía se distingue a Australia con 287 artículos (8,7\%), y por último en Asia está República popular China con 282 artículos, equivalente al $8,5 \%$. 
Respecto al continente americano y tal como se señaló, la producción se concentra en un solo país como Estados Unidos, lo siguen Canadá con 164 artículos. En tanto, Brasil es el país latinoamericano con mayor cantidad de publicaciones de la región con 25 artículos, seguidos por México, Colombia y Chile, respectivamente (Tabla 4). En tanto, el idioma mayoritario de las publicaciones es el inglés, concentrando 3.228 escritos, lo que representa un 97,8\% del total.

\section{Tabla 4}

Artículos publicados según continente y país

\begin{tabular}{|c|c|c|c|c|c|c|c|}
\hline $\begin{array}{l}\text { Conti } \\
\text { nente }\end{array}$ & $\begin{array}{c}\mathrm{N}^{\circ} \text { de } \\
\text { artículos }\end{array}$ & País & $\begin{array}{c}\mathrm{N}^{\circ} \mathrm{de} \\
\text { artículos }\end{array}$ & Continente & $\begin{array}{c}\mathrm{N}^{\circ} \mathrm{de} \\
\text { artículos }\end{array}$ & País & $\begin{array}{c}\mathrm{N}^{\circ} \text { de } \\
\text { artículos }\end{array}$ \\
\hline \multirow{13}{*}{ África } & \multirow{13}{*}{128} & Túnez & 30 & & & Estados unidos & 1225 \\
\hline & & Sur África & 26 & & & Canadá & 164 \\
\hline & & Egipto & 24 & & & Brasil & 25 \\
\hline & & Nigeria & 11 & & & México & 18 \\
\hline & & Ghana & 10 & & & Colombia & 14 \\
\hline & & Tanzania & 7 & América & 1.466 & Chile & 10 \\
\hline & & Marruecos & 6 & & & Argentina & 4 \\
\hline & & Uganda & 6 & & & Costa Rica & 2 \\
\hline & & Camerún & 2 & & & Ecuador & 2 \\
\hline & & Kenia & 2 & & & Santa Lucia & 1 \\
\hline & & Libia & 2 & & & Venezuela & 1 \\
\hline & & Malaui & 1 & & & & \\
\hline & & Mauricio & 1 & & & Australia & 287 \\
\hline \multirow{16}{*}{ Asia } & \multirow{16}{*}{985} & & & Oceanía & 383 & Nueva Zelanda & 85 \\
\hline & & China & 282 & & & Austria & 11 \\
\hline & & Taiwán & 134 & & & & \\
\hline & & Malasia & 102 & \multirow{13}{*}{ Europa } & \multirow{13}{*}{1.599} & Inglaterra & 352 \\
\hline & & India & 75 & & & España & 262 \\
\hline & & Sur Corea & 70 & & & Italia & 152 \\
\hline & & Singapur & 57 & & & Francia & 118 \\
\hline & & Líbano & 33 & & & Alemania & 99 \\
\hline & & Pakistán & 33 & & & Holanda & 74 \\
\hline & & Japón & 31 & & & Bélgica & 67 \\
\hline & & Tailandia & 19 & & & Suecia & 60 \\
\hline & & Indonesia & 16 & & & Noruega & 55 \\
\hline & & $\begin{array}{l}\text { Arabia } \\
\text { Saudita }\end{array}$ & 15 & & & Escocia & 45 \\
\hline & & Vietnam & 15 & & & Gales & 39 \\
\hline & & $\begin{array}{l}\text { Emiratos } \\
\text { Árabe }\end{array}$ & 14 & & & Turquía & 38 \\
\hline & & Qatar & 12 & & & Suiza & 35 \\
\hline
\end{tabular}




$\begin{array}{lrlr}\text { Irán } & 11 & \text { Finlandia } & 32 \\ \text { Israel } & 11 & \text { Rusia } & 30 \\ \text { Baréin } & 9 & \text { Portugal } & 23 \\ \text { Kuwait } & 8 & \text { Dinamarca } & 22 \\ \text { Sri Lanka } & 7 & \text { Polonia } & 15 \\ \text { Omán } & 6 & \text { Chipre } & 13 \\ \text { Bangladés } & 5 & \text { Grecia } & 12 \\ \text { Yemen } & 5 & \text { Rumania } & 10 \\ \text { Jordania } & 4 & \text { Irlanda } & 9 \\ \text { Irak } & 3 & \text { Norte Irlanda } & 7 \\ \text { Hong Kong } & 2 & \text { Serbia } & 4 \\ \text { Filipinas } & 2 & \text { Eslovaquia } & 4 \\ \text { Armenia } & 1 & \text { Croacia } & 3 \\ \text { Brunei } & 1 & \text { Rep. Checa } & 3 \\ \text { Palestina } & 1 & \text { Ucrania } & 3 \\ \text { Siria } & 1 & \text { Luxemburgo } & 2 \\ & & \text { Mónaco } & 2 \\ & & \text { Eslovenia } & 2 \\ & & \text { Albania } & 1 \\ & & \text { Bulgaria } & 1 \\ & & \text { Estonia } & 1 \\ & & \text { Islandia } & 1 \\ & & \text { Rep. Kosovo } & 1 \\ & & \text { Lituania } & 1 \\ & & \text { Rep. } & 1 \\ & & \text { Macedonia } & \end{array}$

Fuente: elaboración propia.

\section{Catastro principales autores y co-autorías}

Respecto al número de investigadores que han participado en investigaciones sobre $\mathrm{CA}$ y desempeño financiero ascienden a 5.568 autores. Al analizar los periodos, 189 autores se concentran en el primer período (1988-1998), 892 en el segundo (1999-2008) y 4.829 en el tercero (2009-2019). Al realizar un análisis a nivel de autor, se puede evidenciar que del primer período, 120 autores no continuaron escribiendo sobre el tema y sólo 112 escribieron un artículo. Además, se puede apreciar que 54 autores se encuentran presentes en el primer y segundo período y 32 investigadores publicaron durante los tres períodos.

El autor con mayor número de publicaciones es la académica de la University of Salamanca, Isabel María García Sánchez (Tabla 5), quien registra 23 artículos y cuyos escritos han sido citados en 
528 ocasiones. De estos artículos, el trabajo que más resalta (según número se citas) es el que releva el papel de los accionistas y acreedores, existiendo una amplia gama de interesados en las actitudes de las empresas hacia la sostenibilidad y que el CA juega un rol preponderante en cuanto a las buenas prácticas de responsabilidad social corporativa. El objetivo de la investigación era demostrar la influencia que poseen ciertas características del CA (junta directiva) en el grado de integración de la información presentada por las principales empresas (568) no financieras de 15 países, para el período 2008-2010. Los resultados mostraron que las oportunidades de crecimiento, el tamaño de una empresa y sus órganos de administración, junto con la diversidad de género, son los factores más importantes en la difusión integrada de la información (Frias-Aceituno et al., 2013).

\section{Tabla 5}

Principales autores sobre directorio y desempeño financiero

\begin{tabular}{|c|c|c|c|c|}
\hline Autor & $\begin{array}{c}\mathrm{N}^{\circ} \\
\text { Doc. }\end{array}$ & Citas/ doc. & Índice $H$ & Institución \\
\hline \multicolumn{5}{|c|}{ Principales autores según número de publicaciones } \\
\hline Garcia-Sanchez, IM & 23 & 58,4 & & University of Salamanca \\
\hline Garcia-Meca, E & 18 & 58,9 & & Technical University of Cartagena \\
\hline Dalton, Dr & 16 & 48,4 & & University Newfoundland \\
\hline Filatotchev, I & 16 & 54,3 & & King's College London \\
\hline Huse, $\mathrm{M}$ & 14 & 55,0 & & Norwegian Business School \\
\hline Martinez-Ferrero, J & 14 & 60,5 & 12 & University of Salamanca \\
\hline \multicolumn{5}{|c|}{ Principales autores según número de citas } \\
\hline Zajac, Ej & 8 & 278,0 & & Northwestern University \\
\hline Westphal, Jd & 5 & 271,6 & & University of Michigan \\
\hline Dalton, Dr & 8 & 152,8 & & University Newfoundland \\
\hline Daily, Cm & 1 & 138,2 & & University of Arkansas Little Rock \\
\hline Post, Corinne & 6 & 136,5 & 15 & Lehigh University \\
\hline Huse, Morten & 6 & 105,7 & & Norwegian Business School \\
\hline Masulis, Ronald & 6 & 80,3 & 30 & University of New South Wales Sydney \\
\hline Gul, Ferdinand & 5 & 59,2 & & Deakin University \\
\hline Bednar, Michael k. & 5 & 59,0 & 10 & University of Illinois System \\
\hline Aguilera, Ruth & 7 & 46,9 & 28 & Northeastern University \\
\hline
\end{tabular}

Fuente: elaboración propia.

En segundo lugar, se encuentra la académica Emma García Meca de la Technical University of Cartagena, quien registra 18 escritos con un total de 395 citas. Entre los documentos con mayor 
relevancia, realizó un análisis de 35 estudios que examinan el efecto sobre la gestión de ganancias de los directorios y la estructura de propiedad de las empresas. El objetivo fue determinar si las diferencias en los resultados son atribuibles a los efectos moderadores relacionados con el sistema de gobierno corporativo, la medición de la variable de gobierno o las especificaciones particulares de los modelos de acumulación discrecional. Se estableció que existe un error de muestreo en estudios anteriores sobre la dualidad del CEO (Chief executive officer) o llamado director ejecutivo y la independencia del comité de auditores. Por otro lado, la medición de la variable dependiente, las acumulaciones discrecionales y el sistema de gobierno corporativo moderan la asociación entre la gestión de ganancias y algunas variables de gobierno corporativo (García-Meca y SánchezBallesta, 2009).

Un tercer autor destacado es Dan Dalton de la University Newfoundland, quien registra 16 documentos. De estos, el artículo con mayor citación corresponde a la investigación realizada sobre la teoría de agencia, donde se plantea que esta teoría domina la investigación sobre las tenencias de acciones y las relaciones de desempeño de la empresa, No obstante, estudios anteriores demuestran que no existe tales relaciones. Es decir que no existen vínculos consistentes para el desempeño de la empresa y la equidad del CEO, funcionario, director, institucional o de los accionistas. Se llevó a cabo una serie de meta-análisis de estudios empíricos relevantes sobre el rendimiento de la propiedad. Sin embargo, los meta-análisis brindan pocos ejemplos de relaciones sistemáticas, lo que brinda poco apoyo para la teoría de la agencia (Dalton et al., 2003).

En cuarto lugar destaca Igor Filatotchev de King's College London, con 16 artículos. Su obra más citada, es el estudio realizado en el Reino Unido a 251 ofertas públicas iniciales (OPI), en la cual examina los vínculos entre las características ejecutivas y no ejecutivas, la propiedad de acciones y el rendimiento a corto plazo medido en términos de "underpricing " de la oferta de acciones. En sus conclusiones afirma que existe una fuerte evidencia de los vínculos entre el compromiso financiero de los directores no ejecutivos y la experiencia y el poder de los ejecutivos. Además, destaca que estos factores de gobernanza desarrollados endógenamente pueden ser utilizados estratégicamente por los equipos de OPI para reducir el alcance de la subvaloración (Filatotchev y Bishop, 2002).

En quinto lugar con 14 investigaciones se encuentra Morten Huse de Norwegian Business School. Su principal estudio se encuentra el relacionado a la existencia de mujeres en los CA y su influencia en el desempeño. Se realizó un estudio a 317 empresas noruegas. Los resultados sugieren que 
alcanzar la masa crítica, pasando de una o dos mujeres (algunas fichas) a al menos tres mujeres (minoría constante): permite mejorar el nivel de innovación empresarial. Además, los resultados muestran que la relación entre la masa crítica de mujeres directores y el nivel de innovación empresarial está mediado por junta de tareas estratégicas (Torchia et al., 2011).

Por último, se encuentra Jennifer Martínez-Ferrero de la University of Salamanca con 14 artículos. En su principal artículo, analizó el efecto de la diversidad del CA en cuanto a género, nacionalidad y el desempeño de los bancos. Se trabajó con una muestra de 159 bancos en nueve países entre el 2004-2010. La evidencia empírica muestra que la diversidad de género aumenta el rendimiento bancario, mientras que la diversidad nacional lo inhibe. Muestran que en contextos de entornos normativos más débiles y de protección de los inversores más bajos, la diversidad de los CA tiene menos influencia en el desempeño de los bancos (García-Meca et al., 2015).

Asimismo, el autor con mayor número citas por documento es Edward J. Zajac, Profesor del Kellogg School of Management, Northwestern University, con 278 citas promedio por documento. En segundo lugar se encuentra el académico James Westphal del Stephen M. Ross School of Business de University of Michigan, con 271,6 citas. Lo interesante es que ambos autores trabajan colaborativamente en varios artículos. Al analizar las redes de co-autoría, se consideró sólo aquellos autores que han publicado al menos 8 artículos (King-Domínguez et al., 2020), posibilitando establecer a lo menos cuatro grupos:

- Grupo 1: Krause, R; Semadeni, M; Filatotchev, I; Wright, M; Withers, M y Boivie, S.

- Grupo 2: Zona, F; Zattoni, A y Minichilli, A.

- Grupo 3: Dalton, Dr y Daily, Cm.

- Grupo 4: Martínez-Ferrero, J; García-Meca, E; Consuelo Pucheta-Martínez, M; GarcíaSánchez, IM y Cuadrado-ballesteros, B.

\section{Análisis de Co-citas}

De los 3.302 artículos de CA y desempeño financiero, se pudo comprobar que se utilizaron más de 70.000 referencias. Entre los autores más citados se encuentra David Yermack, en su investigación denominada "Higher market valuation of companies with a small board of directors", ha recibido 2.026 citaciones (Tabla 6). El autor presentó evidencia con relación a las teorías de que los CA compuestas por menores personas son más efectivas. En su investigación 
utilizó la Q de Tobin, y a través de ella logro establecer una asociación inversa entre el tamaño de la junta y el valor de la empresa en una muestra de 452 empresas de Estados Unidos entre los años 1984 y 1991. Uno de los principales hallazgos fue descubrir que las empresas con CA con menor número de integrantes exhiben valores más favorables para las relaciones financieras, y ofrecen incentivos más fuertes para el desempeño del CEO de la compensación y la amenaza de despido (Yermack, 1996).

\section{Tabla 6}

Principales referencias en los artículos de CA y desempeño financiero

\begin{tabular}{lc}
\hline \multicolumn{1}{c}{ Documento } & N $^{\circ}$ de citas \\
\hline Yermack, D. (1996) & 2026 \\
Core, J. E., Holthausen, R. W. y Larcker, D. F. (1999) & 1338 \\
Klein, A. (2002) & 1293 \\
Milliken, F. J., Martins, L. L. (1996) & 1272 \\
Beasley, M. S. (1996) & 1157 \\
Hillman, A. J., Dalziel, T. (2003) & 988 \\
Adams, Renee B., Ferreira, Daniel (2009) & 913 \\
Hermalin, B. E., Weisbach, M. S. (1998) & 875 \\
Agrawal, A., Knoeber, C. R. (1996) & 822 \\
Coles, Jeffrey L.; Daniel, Naveen D.; Naveen, Lalitha (2008) & 773 \\
Fan, Joseph P. H.; Wong, T. J.; Zhang, Tianyu (2007) & 760 \\
Xie, B., Davidson, W. N., DaDalt, P. J. (2003) & 683 \\
Weinstein, M. C., O'Brien, B., Hornberger, J., et ál... (2003) & 681 \\
Mehran, H. (1995) & 652 \\
Johnson, R. A., Greening, D. W. (1999) & 644
\end{tabular}

Fuente: elaboración propia.

También destaca el trabajo de Core, J. E., Holthausen, R. W. y Larcker, D. con su artículo denominado "Corporate governance, chief executive officer compensation, and firm performance", el cual ha sido referenciado en 1.344 ocasiones. Es importante señalar que estos autores publicaron en Journal of Financial Economics, revista que posee un impacto de 7.976 y un cuartil Q1. Es este documento, los autores planteaban que las medidas del CA y la estructura de propiedad explican una cantidad significativa de variación transversal en la compensación del 
CEO. En general, sus resultados sugieren que las empresas con estructuras de gobierno más débiles tienen mayores problemas de agencia; que los CEO de las empresas con mayores problemas de agencia reciben una mayor compensación; y que las empresas con mayores problemas de agencia se desempeñan peor (Core et al., 1999).

Otro artículo con alto número de citas es el de Klein (Audit committee, board of director characteristics, and earnings management). Este estudio examina si las características del comité de auditoría y de la junta están relacionadas con la gestión de ganancias por parte de la empresa. Se encuentra una relación negativa entre la independencia del comité de auditoría y las acumulaciones anormales. También se encuentra una relación negativa entre la independencia del CA y las acumulaciones anormales. Las reducciones en la independencia de la junta o el comité de auditoría van acompañadas de grandes aumentos en las acumulaciones anormales. Los efectos más pronunciados ocurren cuando el CA o el comité de auditoría están compuestos por una minoría de directores externos. Estos resultados sugieren que las juntas estructuradas para ser más independientes del CEO son más efectivas en el monitoreo del proceso de contabilidad financiera corporativa (Klein, 2002).

Otro autor destacado es Milliken y Martins con el artículo "Searching for common threads: Understanding the multiple effects of diversity in organizational groups", donde revisaron las investigaciones de gestión sobre los efectos de diferentes tipos de diversidad en la composición del grupo en varios niveles organizacionales. Es decir, los CA, grupos de alta gerencia y grupos de tareas organizacionales, con la finalidad de evidenciar de patrones comunes. Los autores argumentan que la diversidad en la composición de los grupos organizacionales afecta los resultados como la rotación y el rendimiento a través de su impacto en los procesos afectivos, cognitivos, de comunicación y simbólicos (Milliken y Martins, 1996).

\section{Palabras claves y co-palabras}

El resultado muestra que se han utilizado 5.915 palabras clave, de las cuales 4.129 han sido propuesta por los autores y 1.786 por WoS. Del conjunto, 416 se han aplicado en al menos 10 ocasiones. El concepto con mayor número de menciones es "corporate governance” (1.602), seguido por "directors" (1.133), "firm performance" (981) y "performance” (980). (Véase Tabla 7 se pueden observar las palabras más utilizadas). 


\section{Tabla 7}

Clúster y frecuencia de uso de las palabras clave

\begin{tabular}{|c|c|c|}
\hline \multicolumn{3}{|c|}{ Clúster 1} \\
\hline Governance (474) & Gender diversity (185) & Women directors (82) \\
\hline Board of directors (398) & Gender (146) & Social-responsibility (55) \\
\hline $\begin{array}{l}\text { Financial performance } \\
(368)\end{array}$ & Corporate boards (102) & Board Diversity (54) \\
\hline Impact (339) & Disclosure (91) & Reputation (50) \\
\hline Diversity (207) & $\begin{array}{l}\text { Corporate social-responsibility } \\
\text { (90) }\end{array}$ & Ethnic Diversity (49) \\
\hline Women (191) & $\begin{array}{l}\text { Corporate social responsibility } \\
(85)\end{array}$ & Leadership (47) \\
\hline \multicolumn{3}{|c|}{ Clúster 2} \\
\hline Directors (1133) & Agency (152) & Top management (90) \\
\hline Performance (980) & Power (122) & Consequences (89) \\
\hline Management (417) & Boards of directors (120) & Turnover (83) \\
\hline Boards (256) & Strategy (112) & Behavior (79) \\
\hline Firm (234) & CEO (111) & Decision-making (78) \\
\hline Agency theory (162) & Firms (102) & Model (77) \\
\hline \multicolumn{3}{|c|}{ Clúster 3} \\
\hline $\begin{array}{l}\text { Corporate governance } \\
(1602)\end{array}$ & Compensation (247) & Empirical-analysis (164) \\
\hline Firm performance (981) & Size $(241)$ & CEO compensation (157) \\
\hline Ownership (786) & Agency costs (204) & CEO duality (141) \\
\hline Ownership structure (425) & Executive-compensation (193) & Companies (129) \\
\hline Board composition (395) & Incentives (188) & Agency problems (126) \\
\hline Determinants (385) & Market (166) & Market valuation (114) \\
\hline \multicolumn{3}{|c|}{ Clúster 4} \\
\hline Board (233) & Association (44) & Financial expertise (23) \\
\hline $\begin{array}{l}\text { Earnings management } \\
\text { (197) }\end{array}$ & Audit committees (39) & $\begin{array}{l}\text { Information asymmetry } \\
\text { (23) }\end{array}$ \\
\hline Audit committee (119) & Expertise (33) & Committee (19) \\
\hline Independence (102) & Malaysia (32) & $\begin{array}{l}\text { Director characteristics } \\
\text { (19) }\end{array}$ \\
\hline Information (97) & Fraud (28) & Markets (18) \\
\hline Quality (90) & Accruals (24) & $\begin{array}{l}\text { Corporate-governance } \\
\text { (17) }\end{array}$ \\
\hline \multicolumn{3}{|c|}{ Clúster 5} \\
\hline \multicolumn{3}{|c|}{ Underwriter reputation (7) } \\
\hline IPO (15) & \multicolumn{2}{|c|}{ Certification $(6)$} \\
\hline Initial public offering (10) & \multicolumn{2}{|c|}{ Initial public offerings (IPOS) (6) } \\
\hline Underpricing (10) & \multicolumn{2}{|c|}{ Price (6) } \\
\hline Venture capital (9) & \multirow{2}{*}{\multicolumn{2}{|c|}{ Venture (5) }} \\
\hline Issues (7) & & \\
\hline
\end{tabular}

Fuente: elaboración propia. 
Paralelamente, los resultados de las palabras clave muestran que los términos pueden asociarse en cinco clúster o conglomerados. El primer clúster (azul) incorpora 150 palabras clave, mientras que el segundo (rojo) y tercero (verde) tienen 257 y 293; en el clúster 4 (amarillo) se incluyen 65 y en el último clúster (morado) hay 11 palabras clave. En la Figura 3 están representados los diferentes clústeres. Cada palabra está asociada a un círculo cuyo tamaño es ajustado el número de registros y las líneas que unen cada uno de esos círculos simbolizan los enlaces de los registros.

\section{Figura 3}

Redes de palabras claves

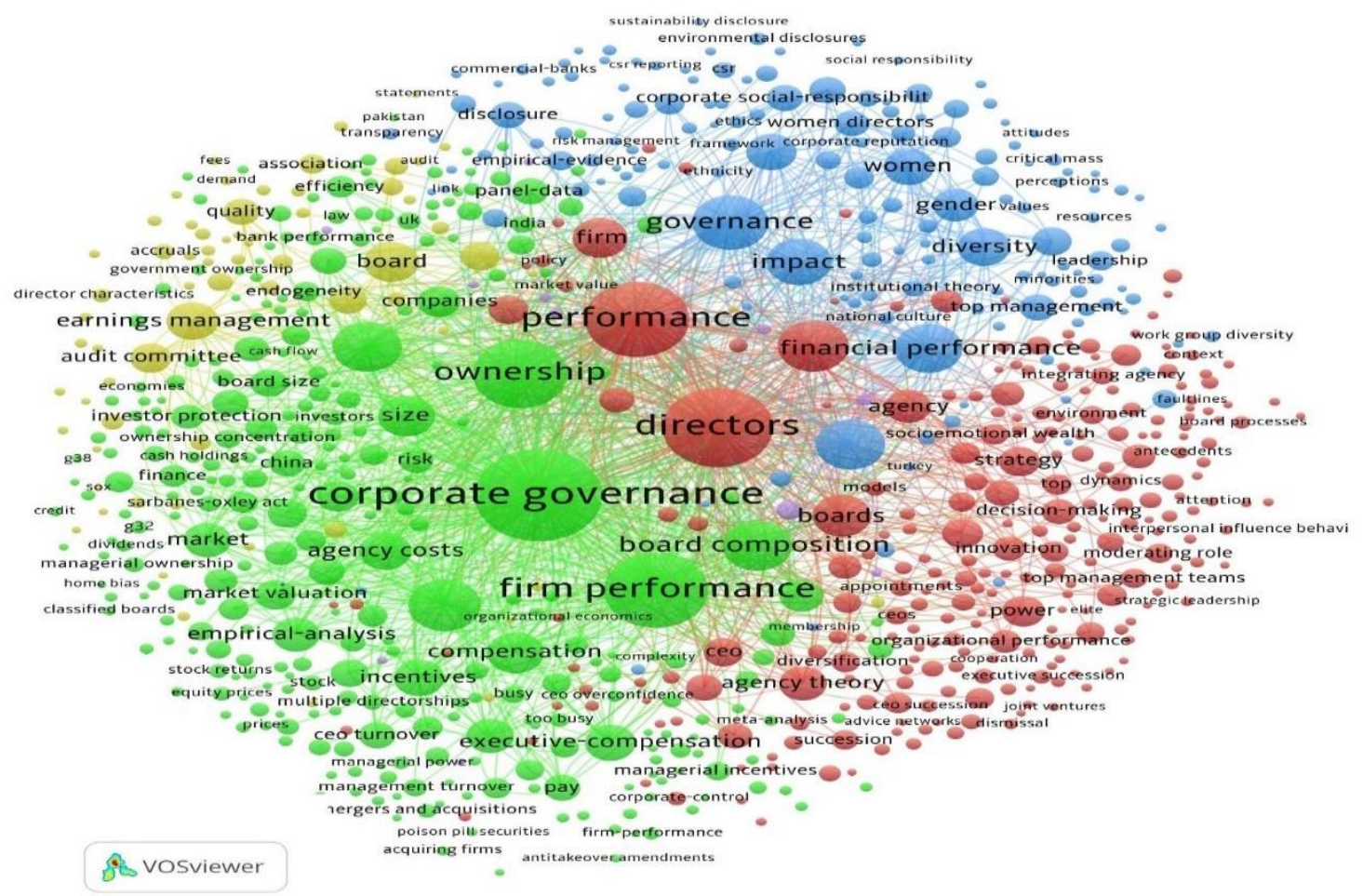

Fuente: elaboración propia a partir de VOSviewer.

\section{Conclusiones}

El CA como órgano máximo de la organización está estrechamente ligado con el desempeño financiero de las empresas. A partir del estudio bibliométrico se puede afirmar que es un tema de alta relevancia y de carácter tendencial, ha preocupado crecientemente a la comunidad científica con predominio de métodos de investigación analítica y experimental. Destacan los estudios con apoyo de métodos econométricos que buscan una relación causa efecto. La palabra clave más citada es gobierno corporativo, seguida de directores y desempeño. Además, se complementa con palabras clave asociadas a estudios de caso territoriales, lo cual indica cierta libertad y poca 
estandarización de conceptos por parte de los editores y sus respectivos comités editoriales. Asimismo, hay un auge y espacio para nuevos estudios en líneas de investigación referidos al impacto en gestión por diversidad, participación de las mujeres y características propias en los CA. También, se observa en forma incipiente estudios ligados a empresas familiares.

La mayor cantidad de artículos se concentran en 13 revistas, donde la mayoría pertenece al Quintil I. Es decir, la productividad se encuentra en la categoría de alto impacto. En cuanto a los autores con mayor número de artículos destaca Isabel María García Sánchez. A su vez, el autor con mayor número citas por documento es Edward J. Zajac y el más citado David Yermack. A nivel internacional, Estados Unidos es el país con mayor productividad. Las redes de colaboración académica internacional son fundamental para escribir a buen nivel y que los casos de economías desarrolladas destacan por sobre los países de economías emergentes.

Es importante enfatizar que esta investigación se ha limitado a la producción científica en la base de datos WoS, excluyendo otras bases también importantes como son Scopus, Scielo y otros tipos de indexaciones, que podrían dar cuenta de nuevos estudios en la temática y avances en el estado de arte, especialmente en Latinoamérica. Asimismo, se definió como parámetro de calidad el concepto de alto impacto, que no necesariamente es consensuada por todo el mundo científico. Siendo esta limitación una opción para abordar en futuras investigaciones.

\section{Referencias}

(1) Al Farooque, O., Buachoom, W., \& Sun, L. (2019). Board, audit committee, ownership and financial performance - emerging trends from Thailand, Pacific Accounting Review, 32(1), 54-81. https://doi.org/10.1108/PAR-10-2018-0079

(2) Core, J. E., Holthausen, R. W., \& Larcker, D. F. (1999). Corporate governance, chief executive officer compensation, and firm performance. Journal of Financial Economics, 51(3), 371-406.

(3) Dalton, D. R., Daily, C. M., Certo, S. T., \& Roengpitya, R. (2003). Meta-analyses of financial performance and equity: Fusion or confusion? Academy of Management Journal, 46(1), 1326. https://doi.org/10.2307/30040673

(4) Fama, E. F., \& Jensen, M. C. (1983). Separation of ownership and control. The journal of law and Economics, 26(2), 301-325. 
(5) Ferreira, C. y De Morán, M. A. (2011). La responsabilidad social corporativa (RSC) en las bases de datos SCOPUS y WOS (Estudio Bibliométrico). Revista EDICIC, 1(4), 141-160.

(6) Filatotchev, I., \& Bishop, K. (2002). Board composition, share Ownership, and “Underpricing” of U.K IPO Firms. Strategic Management Journal, 23(10), 941-955.

(7) Frias-Aceituno, J. V., Rodriguez-Ariza, L., \& Garcia-Sanchez, I. M. (2013). The role of the board in the dissemination of integrated corporate social reporting. Corporate social responsibility and environmental management, 20(4), 219-233. https://doi.org/10.1002/csr.1294

(8) García-Meca, E., \& Sánchez-Ballesta, J. P. (2009). Corporate Governance and Earning management: A meta-analysis. Corporate Governance and International Review, 17(5), 594610. https://doi.org/10.1111/j.1467-8683.2009.00753.x

(9) García-Meca, E., García-Sánchez, I. M., \& Martínez-Ferrero, J. (2015) Board diversity and its effects on bank performance: An international analysis. Journal of Banking \& Finance, 53, 202-214. https://doi.org/10.1016/j.jbankfin.2014.12.002

(10) Huang, C. Y., \& Ho, Y. S. (2011). Historical research on corporate governance: A bibliometric analysis. African Journal of Business Management, 5(2), 276-284. https://doi.org/10.5897/AJBM09.368

(11) King-Domínguez, A., Llinàs-Audet, X. y Améstica-Rivas, L. (2020). Caracterización de la producción científica sobre clasificaciones de universidades. Un estudio bibliométrico desde 1988 a 2018. Formación universitaria, 13(2), 53-62. https://dx.doi.org/10.4067/S071850062020000200053

(12) Klein, A. (2002). Audit committee, board of director characteristics, and earnings management. Journal of Accounting and Economics, 33(3), 375-400. https://doi.org/10.1016/S0165-4101(02)00059-9

(13) Merediz-Solà, I., \& Baraviera, A. (2019). A bibliometric analysis of Bitcoin scientific production. Research in International Business and Finance, 50, 294-305. https://doi.org/10.1016/j.ribaf.2019.06.008

(14) Milliken, F. J., \& Martins, L. L. (1996). Searching for Common Threads: Understanding the Multiple Effects of Diversity in Organizational Groups. Academy of Management review, 21(2), 402-433.

(15) Patel, M. A. (2019). Impact of board structure and firm performance on chief executive's compensation. Asia-Pacifid Management Accounting Journal, 14(2), 185-199. http://dx.doi.org/10.24191/apmaj.v14i2.930 
(16) Torchia, M. T., Calabrò, A., \& Huse, M. (2011). Women directors on Corporate Boards: From tokenism to critical mass. Journal of Business Ethics, 102(2), 299-317.

(17) Van Eck, N. J., \& Waltman, L. (2017). VOSviewer Manual. Leiden.

(18) Yasser, Q. R., Mamun, A. A., \& Rodrigs, M. (2017). Impact of board structure on firm performance: evidence from an emerging economy. Journal of Asia Business Studies, 11(2), 210-228. https://doi.org/10.1108/JABS-06-2015-0067

(19) Yermack, D. (1996). Higher market valuation of companies with a small board of directors. Journal of Financial Economics, 40(2), 185-211. https://doi.org/10.1016/0304405X(95)00844-5

Cómo citar este artículo: Améstica-Rivas, L., King-Domínguez, A., Cruzat, C. y Stuardo, C. (2021). Consejo de administración y desempeño financiero. Un estudio bibliométrico. Tendencias, 22(1), 180-203. https://doi.org/10.22267/rtend.212201.160 